\title{
AS FASES DO TREINAMENTO DE NATAÇÃO INFLUENCIAM OS SUBSTRATOS ENERGÉTICOS, CORTISOL E LEUCÓCITOS
}

\author{
Sueli Cristina Schadeck Zago ${ }^{1,2,3}$, Márcia Regina Pessoa D'Andrade ${ }^{1,2}$, Ana Paula Miskulin Cardoso ${ }^{2,3}$, \\ Cláudio Spinola Najas ${ }^{4,5}$, Cassiano Merussi Neiva ${ }^{6}$, Pedro Balikian Junior ${ }^{7}$, Jair Rodrigues Garcia Junior ${ }^{5}$ \\ ${ }^{1}$ Curso de Farmácia e Bioquímica, ${ }^{2}$ Faculdade de Medicina, ${ }^{3}$ Curso de Enfermagem, ${ }^{4}$ Curso de Fisioterapia e ${ }^{5}$ Curso de Educação \\ Física da UNOESTE, Presidente Prudente, SP; ${ }^{6}$ Programa de Mestrado e Doutorado em Promoção de Saúde da UNIFRAN, Franca, \\ SP; ${ }^{7}$ Departamento de Educação Física da FCT/UNESP, Presidente Prudente, SP.
}

\section{RESUMO}

Os efeitos do exercício físico na função imune têm sido bastante estudados, demonstrando que a intensidade e duração do exercício exercem influência nos parâmetros imunológicos. No entanto, há poucos estudos que comparam exercícios de diferentes intensidades e fases de um programa de treinamento. Assim, o objetivo deste estudo foi verificar as alterações metabólicas, hormonais e imunológicas antes e após sessões de natação em diferentes fases do programa de treinamento. Dezessete nadadores do sexo masculino foram avaliados em três fases do treinamento com diferentes intensidades em cada sessão, com base na máxima velocidade no melhor tempo para a distância, obtidos no melhor desempenho em competição. As sessões foram caracterizadas como de potência anaeróbica (PAN - 90\%), potência aeróbica (PAE - 70\%) e tolerância ao lactato (TLA - 98\%). Amostras de sangue foram coletadas pré e imediatamente após o exercício para contagem de células e determinação da concentração de substratos e de cortisol. Foi utilizada ANOVA para verificar a significância das diferenças $(p<0,05)$. Houve aumento significativo das concentrações de glicose e cortisol após o exercício nas sessões de PAN e PAE. A glutamina aumentou significativamente em PAE e TLA. Os leucócitos aumentaram significativamente após as três diferentes sessões e os linfócitos diminuíram significativamente após as sessões PAE e TLA. Concluindo, foi demonstrado que as alterações nos parâmetros estudados em diferentes fases do treinamento podem causar variações temporárias nas células do sistema imune e comprometer a resistência às infecções comuns e o desempenho do atleta.

Palavras-chave: exercício físico, sistema imune, glutamina, glicose.

\section{THE STAGES OF SWIMMING TRAINING AFFECT ENERGETIC SUBSTRATES, CORTISOL AND LEUKOCYTES}

\begin{abstract}
The effect of physical exercise in immune function has been extensively studied. The intensity and duration of physical exercise have considerable influence in immunologic parameters. However, few studies have compared different exercise intensities in different stages of a physical training program. Thus, the aim of this study was to verify the metabolic, hormonal and immunologic changes before and after acute intermittent swimming exercise, following different stages of training program. Seventeen male swimmers were evaluated in three stages of training. The intensity of the three sessions was $90 \%$ (anaerobic potency PAN), 70\% (aerobic potency - PAE) and 98\% (lactate tolerance - TLA) of the maximal speed from the best time of the distance, resulted from peak performance in competition. Blood samples were collected pre and immediately after exercise for cells counting and measurement of substrates and cortisol concentrations. It was used ANOVA to verify the significance of difference $(p<0.05)$. There was a significant increase of glucose and cortisol post exercise in the PAN and PAE sessions. Glutamine increased significantly in PAE and TLA. Leukocytes increased significantly after the three different sessions and lymphocytes decreased significantly on PAE and TLA. In conclusion, it was demonstrated that changes of the several parameters studied in different stages of training program can cause temporary alterations in immune cells and may compromise resistance to common minor illnesses and athlete performance.
\end{abstract}

Keywords: physical exercise, immune system, glutamine, glucose. 


\section{INTRODUÇÃO}

Informações importantes têm surgido de estudos que relacionam o exercício físico com o sistema imune. O exercício físico pode aumentar algumas das funções e também diminuir outras funções do sistema imune. Enquanto a prática de exercícios de intensidade moderada pode provocar melhora da função imune, comparando com a condição sedentária, exercícios excessivamente prolongados e de moderada-alta intensidade podem suprimir a função imune (PYNE \& GLEESON, 1998; COSTA ROSA \& VAISBERG, 2002). Muitos estudos têm relatado que algumas das funções do sistema imune são temporariamente suprimidas após sessões únicas de exercício prolongado de modera-alta intensidade (NIEMAN, 1994; ROWBOTOTTOM et al., 1995; NAVALTA et al., 2009). Como exemplo, temos atletas que praticam treinamento de endurance durante períodos de semanas e se mostram mais susceptíveis a contrair infecções leves (NIEMAN, 1994; COSTA ROSA \& VAISBERG, 2002; NATALE et al., 2003).

Sessão aguda de exercício de endurance resulta em uma redistribuição relativamente grande de leucócitos entre o sangue e outros tecidos, bem como aumento das citocinas circulantes (COSTA ROSA \& BATISTA JUNIOR, 2005). O número de leucócitos circulantes e sua capacidade funcional podem ser diminuídos por repetidas sessões de exercícios prolongados e de intensidade moderada-alta. A razão para isso, provavelmente, está relacionada com o aumento da concentração de hormônios do estresse durante o exercício (SHINKAl et al., 1996; PEDERSEN et al., 1997) e a chegada à circulação de leucócitos imaturos, saídos da medula óssea (RISOY et al., 2003). Diminuição na concentração sanguínea de glutamina também parece ser possível causa da imunossupressão associada com o treinamento prolongado e de intensidade moderada-alta, apesar das evidências sobre essa causa serem menos convincentes (MacKINNON \& HOOPER, 1996; KOYAMA et al., 1998; AGOSTINI \& BIOLO, 2010). Além disso, alguns estudos mostram que vários indicadores da função de leucócitos são sensíveis ao aumento da carga de treinamento em atletas altamente treinados (GLEESON et al., 1995; PEIJIE et al., 2003). Mesmo ao longo de períodos relativamente curtos (1-3 semanas) de treinamento intenso, têm sido observadas significativas reduções na função de neutrófilos, proliferação de linfócitos e no número de células T produtoras de IFN- $y$ na circulação (GLEESON et al., 1995). Por exemplo, sessões intensas de exercício de endurance $\left(70-85 \% \quad \mathrm{VO}_{2}\right.$ máx $)$ induzem perturbação bifásica da contagem de leucócitos na circulação. Imediatamente após o exercício, o número total de leucócitos aumenta $50-100 \%$, incluindo todos os tipos de leucócitos. Dentro de 30 minutos de recuperação, a contagem de linfócitos começa a diminuir abaixo de $30-60 \%$ do normal, permanecendo baixa durante 3 a 6 horas. No entanto, após exercícios de intensidade moderada, e.g. $50 \%$ do $\mathrm{VO}_{2}$ máx, a contagem de linfócitos não diminui durante o período de recuperação (GLEESON et al., 1995).

Neste sentido, vários estudos têm focado no impacto do exercício em parâmetros do sistema imune após sessões agudas de exercícios de moderada-alta intensidade. Entretanto, não há resultados disponíveis que comparam exercícios de diferentes intensidades em diferentes estágios de um programa de treinamento de nadadores competitivos. Por isso, o objetivo deste estudo foi verificar as alterações em parâmetros metabólicos, hormonais e imunológicos antes e após exercício de natação intermitente e agudo ao longo de diferentes estágios do programa de treinamento, para caracterizar os possíveis prejuízos do exercício intenso no desempenho. 


\section{MÉTODOS}

Sujeitos

Dezessete nadadores do sexo masculino, com idade média de 18,4 $\pm 1,9$ anos, participaram deste estudo. $O$ protocolo estava em conformidade com preceitos éticos e foi avaliado e aprovado pelo Comitê de Ética em Pesquisa da Universidade do Oeste Paulista. Os sujeitos leram e assinaram um termo de consentimento livre e esclarecido para participaram do estudo.

\section{Protocolo de treinamento}

O protocolo de treinamento consistiu de três sessões realizadas em dias diferentes, sempre no período da manhã (08h00). Foi realizado um aquecimento na piscina com nado livre e exercícios de perna no início de cada sessão de treinamento. Na série principal da sessão, a intensidade foi determinada com base na máxima velocidade para o melhor tempo na distância (MVMTD), um parâmetro obtido diretamente no momento do melhor desempenho em competições e de fácil controle durante as sessões de treinamento. A primeira sessão de treinamento analisada foi caracterizada como de potência anaeróbica (PAN), realizada na intensidade de $90 \%$ da MVMTD e consistiu de uma série principal com tiros de nado estilo livre (12x50 m, 8x25 m, 1×100 m e 1x50 m). A segunda sessão foi caracterizada como de potência aeróbica (PAE), realizada na intensidade de $70 \%$ da MVMTD e consistiu de uma série principal com tiros de nado estilo livre $(4 \times 400 \mathrm{~m}$ e 1x200 m). A terceira sessão foi caracterizada como de tolerância ao lactato (TLA), realizada na intensidade de $98 \%$ da MVMTD e consistiu de uma série principal com tiros de nado estilo livre (4x100 m, 1×200 m, 2x50 m e 1×200 m). O intervalo entre cada uma das três sessões/avaliações foi de um mês.
Análises do sangue

As amostras de sangue venoso foram coletadas em repouso (pré), servindo com índice do efeito do treinamento, e imediatamente após a sessão de treinamento (pós), para analisar o efeitos agudo do exercício. Em cada estágio do treinamento, uma sessão foi selecionada para coleta do sangue e análises. A concentração de glicose foi determinada usando o método da glicose oxidase (automated Opera, Bayer, USA), a concentração de lactato usando o método da lactato oxidase (YSI Stat Plus, Yellow Springs Instruments, USA) e a concentração de glutamina usando o método enzimático colorimétrico (LUND, 1974), todos usando espectrofotometria. A concentração de cortisol foi determinada usando $O$ método de quimiluminescência (authomatized DPC, Himmulite, USA) e o número de células brancas foi determinado usando analisador automático (CELLDYN 1400, Abbott, USA).

Análise estatística

A significância estatística das diferenças foi determinada usando ANOVA two way e teste de Tuckey para amostras pareadas, sendo o nível de significância estabelecido em $p<0,05$.

\section{RESULTADOS}

A concentração de lactato imediatamente após o exercício aumentou significativamente em todas as sessões de treinamento, sendo observado o maior aumento na sessão TLA, de $1,16 \pm 0,97$ para $8,01 \pm 0,78 \mathrm{mmol} / \mathrm{L} \quad(p<0,05)$. Também houve diferença significativa pós exercício na comparação entre a sessão de treinamento TLA e as sessões PAN e PAE. A concentração de glicose aumentou significativamente comparando o pré com o pós exercício nas sessões de treinamento PAN e PAE $(p<0,05)$, mas não houve diferenças significativas entre as sessões de treinamento. A concentração 
de glutamina aumentou significativamente pós exercício nas sessões de treinamento PAE e TLA.

A concentração de cortisol aumentou

significativamente pós exercício nas sessões de treinamento $\operatorname{PAE}$ e TLA $(p<0,05)$, porém não houve diferenças significativas entre as sessões de treinamento (Tabela 1).

Tabela 1. Concentrações de substratos e cortisol dos nadadores $(n=17)$ pré e pós as sessões de treinamento em diferentes intensidades.

\begin{tabular}{|c|c|c|c|c|c|c|}
\hline & \multicolumn{2}{|c|}{ Potência Anaeróbica } & \multicolumn{2}{|c|}{ Potência Aeróbica } & \multicolumn{2}{|c|}{ Tolerância ao Lactato } \\
\hline & Pré & Pós & Pré & Pós & Pré & Pós \\
\hline $\begin{array}{l}\text { Lactato } \\
\text { (mmol/L) }\end{array}$ & $1,04 \pm 0,22$ & $6,76 \pm 1,67$ * a & $0,76 \pm 0,22$ & $5,57 \pm 2,08$ *b & $1,16 \pm 0,97$ & $8,01 \pm 0,78 * c$ \\
\hline $\begin{array}{l}\text { Glicose } \\
(\mathrm{mg} / \mathrm{dL})\end{array}$ & $84,7 \pm 10,2$ & $104,8 \pm 18,9$ * & $86,1 \pm 14,2$ & $111,3 \pm 29,3$ * & $81,7 \pm 12,2$ & $94,3 \pm 15,4$ \\
\hline $\begin{array}{l}\text { Glutamina } \\
(\mathrm{mmol} / \mathrm{L})\end{array}$ & $1,41 \pm 0,65$ & $1,26 \pm 0,25$ & $0,90 \pm 0,34$ * & $1,18 \pm 0,37$ & $1,02 \pm 0,41$ * & $1,39 \pm 0,31$ \\
\hline $\begin{array}{l}\text { Cortisol } \\
(\mu \mathrm{g} / \mathrm{dL})\end{array}$ & $29,3 \pm 7,3$ & $31,1 \pm 7,7$ & $23,8 \pm 6,6$ & $31,7 \pm 9,1$ * & $25,8 \pm 6,1$ & $39,5 \pm 12,9$ * \\
\hline
\end{tabular}

* $p<0,05$ comparando com a condição pré na mesma intensidade de treinamento.

${ }^{a}$ Letras diferentes na mesma linha indicam diferença $(p<0,05)$ entre as intensidades de treinamento.

A análise do número de células do sistema immune demonstrou alterações significativas no total de leucócitos. A contagem de leucócitos estava significativamente aumentada pós exercício nas sessões de treinamento PAE e TLA $(p<0,05)$ (Figura 1). Analisando os linfócitos, não foram observadas diferenças significativas nas diferentes sessões de treinamento (Figura 2).

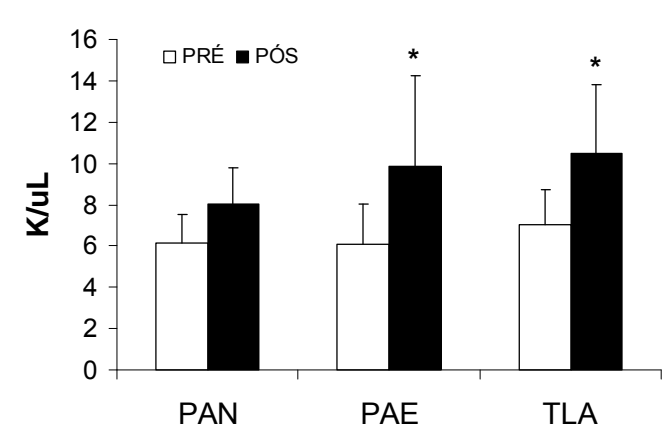

Figura 1. Contagem de leucócitos dos nadadores $(n=17)$ pré e pós as sessões de treinamento de potência anaeróbica (PAN), potência aeróbica (PAE) e tolerância ao lactato (TLA). Os dados são apresentados como média \pm DP. ${ }^{*} p<0,05$ comparando com a condição pré na mesma intensidade de treinamento.

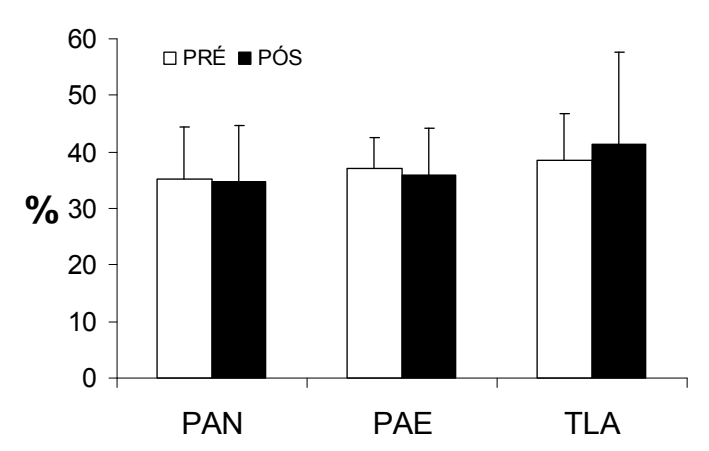

Figura 2. Contagem dos linfócitos dos nadadores $(n=17)$ pré e pós as sessões de treinamento de potência anaeróbica (PAN), potência aeróbica (PAE) e tolerância ao lactato (TLA). Os dados são apresentados como média $\pm \mathrm{DP}$. Não houve diferenças significativas entre os valores pré e pós ou entre as intensidades de treinamento.

\section{DISCUSSÃO}

Este estudo analisou as alterações fisiológicas após a realização de exercício intermitente de natação para determinar as consequências das diferentes intensidades de exercícios em diferentes estágios de treinamento. São apresentadas resultados sobre as alterações nos parâmetros metabólicos, hormonais e 
imunológicos em três diferentes sessões de treinamento.

Demonstramos o aumento significativo da concentração de lactato imediatamente pós exercício em todas as sessões de treinamento e diferença na concentração de lactato pós exercício entre as sessões de treinamento de tolerância ao lactato (TLA), potência anaeróbica (PAN) e potência aeróbica (PAE). Há fartas evidências na literatura demonstrando que o acúmulo de lactato ocorre tardiamente (em percentuais maiores do $\mathrm{VO}_{2}$ máx), após um período de treinamento de endurance. Em atletas, o nível de esforço que pode ser mantido antes do acúmulo de lactato é um preditor preciso do desempenho em esforços de endurance (PEIJIE et al., 2003).

Também foi demonstrado aumento da concentração de glicose pós exercício nas sessões de treinamento de potência anaeróbica (PAN) e potência aeróbica (PAE). Durante exercícios intensos, os músculos esqueléticos são altamente dependentes do metabolismo da glicose (HOROWITZ et al., 1999) e a captação da glicose sanguínea pelos músculos ativos é proporcional à intensidade do exercício (HELGE et al., 2007). Observamos que o aumento da concentração sanguínea de glicose pós exercício pode, provavelmente, ser resultado do efeito hiperglicêmico dos hormônios contra-regulatórios, tais como o cortisol e as catecolaminas (estas últimas, não analisadas).

O aumento na concentração de cortisol está relacionado com a duração e intensidade do exercício físico. Assim, aumento significativo da concentração sanguínea de cortisol, normalmente, ocorre em exercícios com duração de mais que 20 minutos e intensidade de pelo menos $60 \%$ do $\mathrm{VO}_{2}$ máx, sendo, predominantemente, consequência da elevada taxa de secreção na fase imediata pós exercício. Após o pico de concentração, o cortisol normalmente diminui rapidamente e, dentro de poucas horas, retorna ao valor basal (URHAUSEN et al., 1995). Nossos resultados coincidiram com os de outros estudos (SHINKAI et al., 1996; MENDES et al., 2009), demonstrando aumento na concentração de cortisol, principalmente, na sessão de treinamento de tolerância ao lactato (TLA), a mais intensa.

Por sua vez, a glutamina é um importante substrato para as células do sistema imune (CALDER, 1995; PEDERSEN et al., 1997; KOYAMA et al., 1998; AGOSTINI \& BIOLO, 2010). Exercícios intensos diminuem a concentração sanguínea de glutamina e isto pode estar relacionado com a imunossupressão (MacKINNON \& HOOPER, 1996; KOYAMA et al., 1998; AGOSTINI \& BIOLO, 2010). Parece haver uma correlação inversa entre a duração do exercício de intensidade moderada-alta com a concentração de glutamina (ROWBOTTOM et al., 1995) e ocorre diminuição da concentração de glutamina após uma sessão de exercício prolongado, i.e. corrida de maratona (WALSH et al., 1998) e treinamento com repetidas sessões de exercícios prolongados e de intensidade moderada-alta (MacKINNON \& HOOPER, 1996). Por outro lado, em exercício de intensidade alta, porém de duração curta ou média (menos que 1 h), há resultados sobre inalteração ou pequeno aumento da concentração de glutamina (AGOSTINI \& BIOLO, 2010), como foi observado neste estudo nas sessões treinamento de potência aeróbica (PAE) e de tolerância ao lactato (TLA).

O aumento do número de leucócitos é característico do estresse induzido pelo exercício, como consequência da proliferação de neutrófilos (PEDERSEN et al., 1997; RISOY et al., 2003). Neste estudo, foi observado aumento significativo do total de leucócitos. As alterações do número de células do sistema immune têm sido explicadas como efeito da modulação 
neuroendócrina pela adrenalina, noradrenalina, hormônio do crescimento, endordina e cortisol (PEDERSEN et al., 1997). O aumento do número de células durante e imediatamente após o exercício é mencionado em alguns estudos, sendo mencionado o papel específico das catecolaminas e do hormônio do crescimento, como estimuladores (PEDERSEN et al., 1997). Enquanto o cortisol é mencionado como um imunossupressor da proliferação e função das células do sistema imune no período de recuperação (SHINKAl et al., 1996), período que não foi analisado neste estudo. É interessante notar que não houve diferenças na concentração de cortisol e número de células em repouso (pré) nos diferentes dias analisados, o que representou a condição (efeito crônico do treinamento) dos sujeitos nos três diferentes estágios. Estes resultados podem ser entendidos como ausência de condição de overtraining, visto que são dois indicadores deste problema sofrido por alguns atletas (MacKINNON, 1997; SMITH, 2003; KARGOTICH et al., 2005).

Em estudos com nadadores de elite, as alterações nos leucócitos não se mostraram significativas ao longo de uma temporada de seis meses de competições, porém houve aumento significativo no número de linfócitos e neutrófilos pouco antes da principal competição, nos nadadores que exibiam sintomas da síndrome de overtraining. Entretanto, todas as contagens estavam dentro do intervalo dos valores clínicos normais. O aumento do número de neutrofilos foi atribuído ao efeito tardio e prolongado (>24 h) da sessão de treinamento anterior nos nadadores com overtraining que mantiveram o elevador volume de treinamento no período pré competição, em comparação com os nadadores bem treinados que reduziram o volume de treinamento (MacKINNON, 1997).

A possibilidade de uma sessão de exercício ou programa de treinamento influenciar o eixo neuro-endocrino (COSTA ROSA \& BATISTA JUNIOR, 2005), a concentração de enzimas antioxidants essenciais para função imune (DERESZ et al., 2007), bem como a fagocitose de macrófagos (DONATTO et al., 2008), nunca pode ser descartada, já que, não apenas o desempenho, mas também o estado de saúde (VERRATTI, 2009) estão em jogo. Felizmente, a relação entre o exercício e a função imune tem informações abundantes na literatura (RANIERI et al., 2009) e a possibilidade de regulação e adaptação ao longo do programa de treinamento (PEDERSEN \& HOFFMAN-GOETZ, 2000) são conhecidas e aplicáveis.

\section{CONCLUSÃO}

Este estudo demonstrou que os diferentes estágios do programa de treinamento causam alterações temporárias em vários dos parâmetros fisiológicos estudados. Períodos de treinamento intenso podem resultar em disfunção duradoura da função imune. Em vários estudos já realizados foi confirmado o efeito agudo e crônico do exercício na função immune, porém, poucos demonstraram a relação entre os diferentes estágios de um programa de treinamento com a função imune e os efeitos prejudiciais do exercício intenso no desempenho de atletas. Apesar dos atletas não demonstrarem deficiência clínica do sistema immune, é importante a atenção a essa possibilidade, pois os efeitos combinados de pequenas alterações em vários parâmetros imunológicos podem comprometer a resistência às infecções leves e o desempenho dos atletas. Este é um importante tópico que deve ser melhor considerado em futuros estudos.

\section{AGRADECIMENTOS}

Os autores agradecem a João Carlos dos Santos pela assistência técnica na coleta e análise do material. Este estudo foi financiado pela UNOESTE. 


\section{CONFLITO DE INTERESSE}

Os autores declaram não haver qualquer potencial conflito de interesse que possa interferir na imparcialidade deste trabalho científico.

\section{REFERÊNCIAS}

Agostini F, Biolo G. Effect of physical activity on glutamine metabolism. Curr Oin Clin Nutr Metab Care. 2010;13(1):58-64. http://dx.doi.org/10.1097/MCO.0b013e328332f946

Calder PC. Fuel utilization by cells of the immune system. Proc Nutr Soc. 1995;54:65-82. http://dx.doi.org/10.1079/PNS19950038

Costa Rosa LFBP, Batista Junior ML. Efeito do treinamento físico como modulador positivo nas alterações no eixo neuroimunoendócrino em indivíduos com insuficiência cardíaca crônica: possível atuação do fator de necrose tumoral-a. Rev Bras Med Esporte. 2005;11(4):238-42.

Costa Rosa LFPB, Vaisberg MW. Influências do exercício na resposta imune. Rev Bras Med Esporte. 2002;8(4):167-72.

Deresz LF, Lazzarotto AR, Manfroi WC, Gaya A, Sprinz E, Oliveira AR et al. O estresse oxidativo e o exercício físico em indivíduos HIV positivo. Rev Bras Med Esporte. 2007;13(4):275-9. http://dx.doi.org/10.1590/S151786922007000400013

Donatto FF, Prestes J, Ferreira CKO, Dias R, Frollini $A B$, Leite $G S$ et al. Efeitos da suplementação de fibras solúveis sobre as células do sistema imune após exercício exaustivo em ratos treinados. Rev Bras Med Esporte. 2008;14(6):528-32.

Gleeson M, Mcdonald WA, Cripps AW, Pyne DB, Clancy RL, Fricker PA. The effect on immunity of long-term intensive training in elite swimmers. Clin Experim Immun. 1995;102:210-6. http://dx.doi.org/10.1111/j.1365-

2249.1995.tb06658.x

Helge JW, Stallknecht B, Tichter EA, Galbo H, Kiens B. Muscle metabolism during graded quadriceps exercise in man. $\mathrm{J}$ Physiol. 2007;581(Pt 3):1247-58. http://dx.doi.org/10.1113/jphysiol.2007.128348

Horowitz JF, Mora-Rodriguez R, Byerley LO, Coyle EF. Substrate metabolism when subjects are fed carbohydrate during exercise. Am J Physiol. 1999;276:E828-E835.

Kargotich S, Rowbottom DG, Keast D, Goodman C, Dawson B, Morton AR. Plasma glutamine changes after high-intensity exercise in elite male swimmers. Res Sports Med. 2005;13(1):7-21. http://dx.doi.org/10.1080/15438620590922040

Koyama K, Kaya M, Hori JTS. Effects of decreased plasma glutamine concentrations on peripheral lymphocyte proliferation in rats. Eur $\mathrm{J}$ Appl Physiol. 1998;77:25-31. http://dx.doi.org/10.1007/s004210050295

Lund $P$. Determination of glutamine with glutaminase and glutamate dehydrogenase. In: Bergmeyer $\mathrm{HU}$ (Ed.). Methods of Enzymatic Analysis. New York: Academic Press, 1974.

Mackinnon LT, Hooper SL. Plasma glutamine and upper respiratory tract infection during intensified training in swimmers. Med Sci Sports Exerc 1996;28:285-90.

http://dx.doi.org/10.1249/00005768-199603000$\underline{00003}$

Mackinnon LT. Immunity in Athletes. Int J Sports Med. 1997;18(suppl.):S62-S68. http://dx.doi.org/10.1055/s-2007-972701

Mendes EL, Brito CJ, Batista ECS, Silva $\mathrm{CHO}$, Paula SO, Natali AJ. Influência da suplementação de carboidrato na função imune de judocas durante o treinamento. Rev Bras Med Esporte. 2009;15(1):58-61.

http://dx.doi.org/10.1590/S1517$\underline{86922009000100013}$

Natale VM, Brenner IK, Moldoveanu AI, Vasiliou $P$, Shek P, Shepard RJ. Effects of three different types of exercise on blood leukocyte count during and following exercise. São Paulo Med J. 2003;121(1):9-14.

http://dx.doi.org/10.1590/S1516-

31802003000100003

Navalta JW, McFarlin BK, Lyons TS, Faircloth JC, Bacon NT, Callahan ZJ. Exercise-induced lymphocyte apoptosis attributable to cycle ergometer exercise in endurance-trained individuals. Appl Physol Nutr Metab. 2009;34(4):603-8. http://dx.doi.org/10.1139/H09$\underline{018}$

Nieman DC. Exercise, upper respiratory tract infection, and the immune system. Med Sci Sports Exerc 1994;26:128-39. http://dx.doi.org/10.1249/00005768-19940200000002

Pedersen BK, Bruunsgaard H, Klokker M, Kappel $M$, MacLean DA, Nielsen $\mathrm{HB}$ et al. Exerciseinduced immunomodulation - possible roles of neuroendocrine and metabolic factors. Int $\mathrm{J}$ Sports Med 1997;18(Suppl I):2-7. http://dx.doi.org/10.1055/s-2007-972695 
Pedersen BK, Hoffman-Goetz L. Exercise and the Immune System: regulation, integration, and adaptation. Physiol Rev. 2000;80(3):1055-81.

Peijie C, Hongwu L, Fengpeng X, Jie R, Jie Z. Heavy load exercise induced dysfunction of immunity and neuroendocrine responses in rats. Life Sci. 2003;72:2255-62. http://dx.doi.org/10.1016/S0024-3205(03)00115-2

Pyne DB, Gleeson M. Effects of intensive exercise training on immunity in athletes. Int J Sports Med 1998:19(Suppl

3):S183-S191. http://dx.doi.org/10.1055/s-2007-971991

Ranieri M, Megna M, Lancioni GE, Jirillo E, Amico AP, Nardulli $M$ et al. Physical exercise and the immune system. Int Immunopathol Pharmacol. 2009;22(3 suppl):29-32.

Risoy BA, Raastad T, Hallen J, Knut TL, Baeverfjord K, Kravdal A et al. Delayed leukocytosis after hard strength and endurance exercise: aspects of regulatory mechanisms. BMC Physiol. 2003;3(1):14. http://dx.doi.org/10.1186/1472-6793-3-14

Rowbottom DG, Keast D, Goodman C, Morton AR. The haematological, biochemical and immunological profile of athletes suffering from the overtraining syndrome. Eur J Appl Physiol 1995;70:502-9.

http://dx.doi.org/10.1007/BF00634379

Shinkai S, Watanabe $S$, Asai $H$, Shek $P$ N. Cortisol response to exercise and post- exercise suppression of blood lymphocyte subset counts. Int J Sports Med. 1996;17 (8):597-603. http://dx.doi.org/10.1055/s-2007-972901

Smith DJ. A framework for understanding the training process leading to elite performance. Sports Med. 2003;33(15):1103-26. http://dx.doi.org/10.2165/00007256-200333150$\underline{00003}$

Urhausen A, Gabriel H, Kindermann W. Blood hormones as markers of training stress and overtraining. Sports Med. 1995;20(4):251-76. http://dx.doi.org/10.2165/00007256-199520040$\underline{00004}$

Verratti V. Neuroimmune biology of physical exercise. J Biol Regul Homeost Agents. 2009;23(3):203-6.

Walsh NP, Blannin AK, Clark LC, Robson PJ, Gleeson M. The effects of high-intensity intermittent exercise on the plasma concentrations of glutamine and organic acids. Eur $\mathrm{J}$ Appl Physiol. 1998;77:434-8. http://dx.doi.org/10.1007/s004210050356 